\title{
Influence of a lower leg brace on traction force in healthy and ruptured Achilles tendons
}

\author{
Olof Sandberg, Ida Dånmark, Pernilla Eliasson and Per Aspenberg
}

\section{Linköping University Post Print}

\section{Tweet}

N.B.: When citing this work, cite the original article.

Original Publication:

Olof Sandberg, Ida Dånmark, Pernilla Eliasson and Per Aspenberg, Influence of a lower leg brace on traction force in healthy and ruptured Achilles tendons, 2015, MLTJ Muscles, Ligaments and Tendons Journal, (5), 2, 63-67.

http://dx.doi.org/10.11138/mltj/2015.5.2.063

Copyright: CIC Edizioni Internazionali

http://www.mltj.org/

Postprint available at: Linköping University Electronic Press

http://urn.kb.se/resolve?urn=urn:nbn:se:liu:diva-125335 


\section{Influence of a lower leg brace on traction force in healthy and ruptured Achilles tendons}

\author{
Olof Helge Sandberg \\ Ida Dånmark \\ Pernilla Eliasson \\ Per Aspenberg
}

Department of Linköping University, Sweden

Corresponding author:

Olof Helge Sandberg

Department of Linköping University

Hälsouniversitetet

58115 Linköping, Sweden

E-mail: olof.sandberg@liu.se

\begin{abstract}
Background: we investigated how ruptured Achilles tendons are loaded in a brace. There is an ongoing discussion whether patients should be recommended to bear weight on the injured limb. However, little is known about the effects of bracing on tensional loading of the healing Achilles tendon: it is uncertain if load-bearing actually stresses the Achilles tendon inside a brace. Methods: we measured plantar flexion moment inside the brace, in order to estimate tensional loading of the tendon, by use of an insole with pressure transducers.

Results: after wearing the brace for 1 hour, young healthy individuals reduced their maximum flexion moment during gait by half. Patients with Achilles tendon rupture showed no measurable flexion moment during gait with the brace, 4 or 7 weeks after injury. Only when specifically instructed, they could produce a considerable plantar flexion moment. We noted that gait speed with the brace at 4 weeks correlated with a heel-raise functional test at 1 year: the higher the spontaneous gait speed, the less the functional difference between the injured and the uninjured leg $\left(r^{2}=0.68 ; p=0.002\right)$.

Conclusion: the correlation with gait speed suggests that the patients' general physical aptness has an impact on the end result.
\end{abstract}

KEY WORDS: Achilles tendon rupture, bracing, immobilization, loading, flexion moment.

\section{Introduction}

A lower leg cast or brace is routinely used in the treatment of Achilles tendon ruptures, operated or not. It is thought to have two functions: to reduce tension due to dorsiflexion and to reduce active loading of the Achilles tendon. Regarding the treatment of Achilles tendon ruptures, it is often discussed when and how "loading" should be allowed; the term loading then referring to weight-bearing. However, it is possible to bear full weight on a leg without activating the calf muscles, and thus without tensional loading of the tendon. The tendon is loaded by muscle contraction, and it is not well known how that is influenced by bracing.

There is a large inter-patient variation in the stiffness of the Achilles tendon at 7 weeks after injury ${ }^{1}$, the time-point when older treatment regimens start loading the tendon. Moreover, the stiffness at this time-point can partly predict the functional result one year later. Because mechanical loading is a strong stimulator of tendon healing, a possible explanation could be that involuntary muscle activity during bracing shows a great variability, and that this activity influences both early stiffness and late results. Therefore we have measured the plantar flexion moment produced over the ankle joint during bracing, as well as walking speed, of healthy subjects and of patients with Achilles tendon rupture at two time points during the bracing period, and after 12 months. In conjecture with this, we measured the functional outcome in terms of ability to perform heal rises at 12 months. We then analyzed these results to see if any relationship between behavior during the bracing period, and result at 12 months post-op could be found.

\section{Materials and methods}

\section{Healthy volunteers}

Twenty healthy physiotherapy students (10 men and 10 women) were examined during gait in normal sports shoes using the insole measuring device as described below. Thereafter they wore the removable foam walker boot depicted in Figure 1, Rebound Air Walker (Össur, California, USA), on their right leg for 1 hour, walking at least 2500 steps, before measuring moment during normal gait during $3^{*} 1$ min., as described below. 


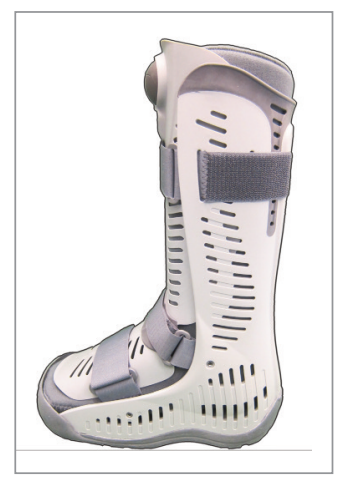

Figure 1. Orthosis worn by patients and healthy volunteers in the study.

\section{Patients and surgery}

Eleven patients volunteered to participate in the study (age 22 to 47 years, 10 males, 1 female). Surgery was performed using a dorso-medial approach and an open technique. All patients were operated on in local anaesthesia, using mepivacaine hydrochloride (Carbocaine) with adrenaline. The proximal and distal tendon stubs were adapted using a resorbable suture (Vicryl ${ }^{\circledR}$ Size 0 ) and using a single loop, modified Kessler technique. During surgery, 4 Tantalum metal markers, with a diameter of $0.8 \mathrm{~mm}$, were placed within the tendon substance. Two markers were placed in the proximal part of the tendon and two were placed in the distal part. These were used for another study, so far not reported. The paratenon was closed thoroughly and the skin was sutured with a resorbable intracutaneous suture (Monocryl 3-0).

After surgery, a short leg cast was applied, with the foot in equinus position. The cast was removed after 2 weeks and replaced by the removable foam walker boot in Figure 1 for another 5 weeks. Patients were provided with three heel wedges in the beginning, and removed one heel wedge every week, i.e. the foot was in neutral position without any heel wedge after 5 weeks. After 7 weeks in total ( 5 weeks in a foam walker), the walker was removed. Full weightbearing was allowed as tolerated from the beginning. For the two first weeks, as long as the patients had a cast in an equinus position, they could only bear weight on the frontal part of the cast. During the period with the foam walker, patients visited a specially trained physiotherapist twice, and were instructed to remove the brace twice a day and perform unloaded ankle motion exercises. Between 7 and 9 weeks, patients gradually ceased using crutches and increased activity according to written instructions, aiming at return to sports after 5 months.

The patients volunteered to measure flexion moment while wearing the brace, 4 and 7 weeks after injury (immediately before brace removal) and during unbraced gait at 52 weeks as described below. A deviation of 3 days from the exact time points was allowed.

\section{Measurements}

Anin-sole plantar pressure measuring device was placed within the brace under the foot and another one in the shoe of the uninjured leg, so that the force from both feet was measured simultaneously. The sole registered loading at around 900 different measuring points (Fscan, Tekscan, Boston, USA). The force at each point multiplied by its known distance from the axis of rotation yielded the flexion moment at the point, and these were added to produce a total flexion moment. These calculations were done with a custom algorithm made with Matlab R2014a (Mathworks, Massachusetts, USA). Calculation of flexion moments required that the projection of the axis on the insole was defined. For this calibration, patients were asked to relax, sitting in a chair with the brace and its insole on a bathroom balance on the floor, and the lower leg vertical. The investigator then applied a $250 \mathrm{~N}$ vertical load on the patient's knee. The loading was considered to produce no moment, and the rotational axis was defined as the one which produced a nil value for moment from the insole (Fig. 2).

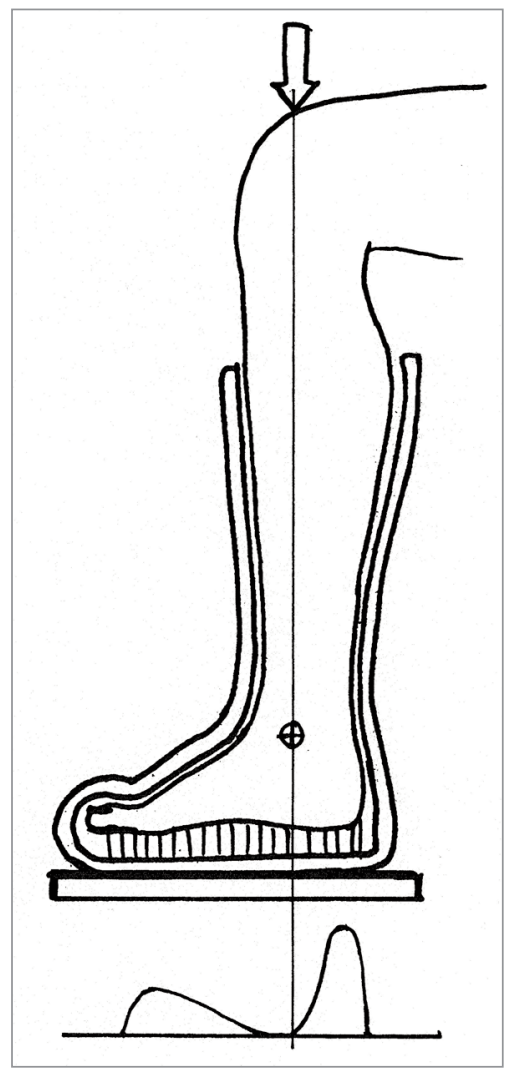

Figure 2. Determination of where the axis of rotation projects on the measuring sole. The patient was asked to relax, and an external vertical force was applied (arrow). The axis was defined by where the flexion and extension moments were equal. The curve below symbolizes the distribution of the moment between measuring points in the sole. The axis of rotation was used to calculate moment from the force measurements. 
For the patients with Achilles tendon rupture, this procedure was performed on both legs. The axis on the braced side tended to get a more posterior location than in the uninjured leg, probably because of protective over-activation of dorsal flexors. Therefore, the position of the axis in the uninjured leg was applied for both sides.

Time sequences were registered. The insole was connected to a computer via a cable, so the test subject walked in a figure of eight, about 10 meters in either direction. The peak moment for all steps was registered, and the lowest third of the peaks (considered corresponding to turning around) was excluded. The mean of the remaining peaks was used for analysis. The maximum tendon force was estimated using the measured moment and a moment arm from the center of the talar trochlea and the tendon, as measured on CT scans.

For the patients, tendon stiffness at 7, 19 and 52 weeks was measured using the distance between tantalum beads injected into the proximal and distal tendon. The patient resisted 2 different dorsal flexion moments, applied by different weights. The change in distance between the beads was measured by radiostereometry (RSA). The transverse area of the tendon was measured by CT. These data were then used to calculate an estimate for modulus of elasticity. The method has been previously described in detail $^{2}$. As an extension to those measurements, patients in the current study were also asked to resist a constant dorsal-flexing moment during 3 minutes. At the 52 week time point, a heal rise index (HRI) was measured $^{1}$. HRI is the number of one-legged heal rises a patient can make, multiplied by the maximum heel-rise height. This value was then normalized to the healthy side, and expressed in percent.

The study was approved by the Linköping Regional Board for Ethics in Research. Informed consent was obtained and the rights of subjects were protected, in accordance with the MLTJ guidelines ${ }^{3}$.

\section{Results}

In the healthy young students, 1 hour of bracing reduced the flexion moment during gait by $48 \%$ as compared with the same foot without brace (SD 16, Tab. 1 ). In contrast, only minuscule moments could be measured in the tendon rupture patients at 4 weeks (mean $0.28 \mathrm{Nm}$ ) and 7 weeks (mean $0.29 \mathrm{Nm}$ ). However, the patients were able to produce a constant moment of $11 \mathrm{Nm}$ (range 5 to 15) during 3 minutes when asked to resist loading during the RSA measurements at week 7. All patient data are given in Table 2 .

Walking speed, 4 weeks after the Achilles tendon rupture, tended to be reduced and correlated with the $\mathrm{HRI}$ at 12 months ( $\mathrm{r}^{2}=0.68, \mathrm{p}=0.002$, Fig. 3$)$. At 7 weeks, gait speed seemed to have normalized and was less strongly correlated to $\mathrm{HRI}$ at one year $\left(\mathrm{r}^{2}=\right.$ $0.30, p=0.08)$.

Table 1. Healthy volunteers force measurement results.

\begin{tabular}{lll}
\hline & Normal gait $(\mathrm{n}=20)$ & With brace $(\mathrm{n}=20)$ \\
\hline Average $(\mathrm{Nm})$ & 81.5 & 42.9 \\
SD $(\mathrm{Nm})$ & 25.4 & 18.5 \\
\hline
\end{tabular}

Table 2. Achilles tendon rupture patient results from RSA, moment measurement, and HRI.

\begin{tabular}{|c|c|c|c|c|c|c|c|c|c|c|}
\hline \multirow[t]{2}{*}{ Patient } & \multicolumn{3}{|c|}{ E-modulus at RSA (MPa) } & \multicolumn{3}{|c|}{$\begin{array}{l}\text { Peak moment on ruptured side } \\
\text { normalized to healthy side (\%) }\end{array}$} & \multicolumn{3}{|c|}{ Steps per second (1/s) } & \multirow{2}{*}{$\begin{array}{l}\mathrm{HRI}(\%) \\
52 \mathrm{w}\end{array}$} \\
\hline & $7 w$ & $19 w$ & $52 w$ & $4 w$ & $7 w$ & $52 w$ & $4 w$ & $7 w$ & $52 w$ & \\
\hline 1 & & & & 6,4 & 2,5 & 89 & 0.85 & 0.85 & 0.78 & 0.93 \\
\hline 2 & 83 & 76 & 147 & -1.2 & -1.6 & 85 & 0.72 & 0.72 & 0.78 & 0.07 \\
\hline 3 & 113 & 77 & 250 & 0.8 & 0.3 & 89 & 0.76 & 0.73 & 0.79 & 0.43 \\
\hline 4 & 70 & 28 & 238 & -2.5 & -1.1 & 56 & 0.74 & 0.79 & 0.79 & 0.16 \\
\hline 5 & & 70 & 164 & 0.0 & 1.4 & 83 & 0.73 & 0.73 & 0.75 & 0.19 \\
\hline 6 & 45 & 76 & 266 & 0.6 & 8.3 & 122 & 0.75 & 0.70 & 0.75 & 0.36 \\
\hline 7 & 53 & 112 & 365 & -0.1 & -0.5 & 104 & 0.86 & 0.87 & 0.91 & 0.71 \\
\hline 8 & 122 & 76 & & -2.3 & 2.0 & 107 & 0.84 & 0.79 & 0.79 & 0.55 \\
\hline 9 & 53 & 14 & 180 & 0.4 & 0.4 & 122 & 0.74 & 0.77 & 0.92 & 0.45 \\
\hline 10 & 63 & 100 & 70 & 1.3 & 6.9 & 110 & 0.83 & 0.82 & 0.85 & 0.88 \\
\hline 11 & 45 & & 294 & 0.1 & -0.1 & 116 & 0.83 & 0.77 & 0.75 & 1.27 \\
\hline Mean & 72 & 70 & 219 & 0.32 & 1.7 & 98 & 0.79 & 0.78 & 0.81 & 0.55 \\
\hline SD & 27 & 29 & 83 & 2.25 & 3.0 & 19 & 0.05 & 0.05 & 0.06 & 0.35 \\
\hline
\end{tabular}




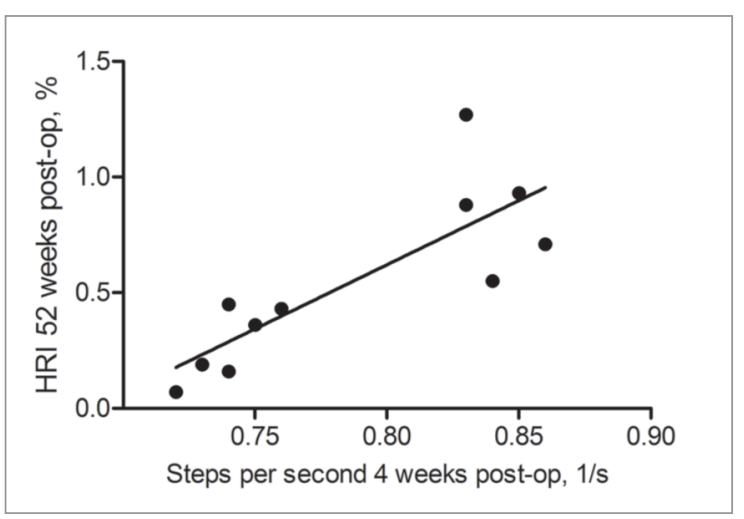

Figure 3. Heel rise index (HRI) 52 weeks post-op correlated with walking speed at 4 weeks post-op. $r^{2}=0.68, p=$ 0.002 .

We were unable to find correlations between spontaneous plantar flexion moments during gait and the $\mathrm{HRI}$ or tendon modulus measured with the RSA method.

Some patients reported that they had removed the brace and walked indoors without it, in spite of instructions not to do so. We had the impression that these patients showed a higher walking speed. One of these patients was so used to walking without brace that he suggested to repeat the measurements without, which we did. His braced maximum flexion moment was $8.8 \mathrm{Nm}$ (8\% of the uninjured contralateral leg). With an ordinary shoe, it was $23 \mathrm{Nm}$ (25\% of the contralateral).

\section{Discussion}

We found that Achilles tendon rupture patients walking with a brace produced very small moments over the ankle rotational axis, indicating a minimal stress in the Achilles tendon. Furthermore, there was a correlation between walking speed after 4 weeks in a brace and ability to perform heal rises at 52 weeks post-op.

Bracing reduced the tendon force in healthy subjects, but in spite of the lack of a need for active plantar flexion inside the brace, they still activated their calf muscles to a considerable degree, showing that bracing in itself is not sufficient to unload the tendon in healthy individuals. In the rupture patients, the healing tendon was able to resist a considerable force at 7 weeks, as shown during the RSA examination. However, the patients seemed not to activate their calf muscles while walking with the brace. Although bracing only moderately reduces muscle force production in the healthy, it seemed to allow almost complete relaxation of the muscles during healing after injury: the maximum moment measured during walking at 7 weeks after injury corresponded to $22 \%$ of the constant voluntary moment produced during 3 minutes at the radiographic examination at the same visit.
A strength of this study is that forces were measured inside the brace, as it is not possible to measure ankle moments in a brace during conventional gait analysis. A weakness is that the calculation of flexion moments required a projection of the rotational axis on the insole. This might be a cause of error. However, we simulated a systematic misplacement of the axis by as much as $10 \mathrm{~mm}$ (which is more than can be expected) in the direction that produces a falsely high moment, and this still only increased the moment to less than half of what was voluntarily produced during the RSA examination. As we measured moment, and not tendon force, we can't exclude that the patients with Achilles rupture contracted their other dorsal flexors, leading to falsely high estimations of Achilles tendon stress. Patients with an Achilles tendon rupture tend to compensate the loss of soleus activation during isometric plantar flexion by a higher activation of flexor halluces longus ${ }^{4}$. Thus, even though our measurement suggested a small plantar flexion moment, they do not imply that any force was produced by the triceps surae.

In a previous randomized trial, patients were encouraged to load their Achilles tendon in a controlled way twice daily during the bracing period, up to a moment around $23 \mathrm{Nm}$. This lead to an increased stiffness of the healing Achilles tendon at 19 and 52 weeks ${ }^{1}$. The present results confirm that the tendons in the control group of that study (continuous bracing) did not experience forces of the same magnitude as the active loading group. Therefore it can be recommended to briefly, but regularly, remove the brace during the healing period for some kind of mechanical stimulation.

Our finding that immobilization removes roughly half of the torque or moment of the Achilles tendon in healthy subjects corresponds well to other studies. By deriving muscle force from EMG, it has been shown that healthy volunteers during walking, e.g. without a brace, used about $30 \%$ of their maximum voluntary plantar flexor torque. When walking with a brace, these values were reduced to 12 to $21 \%$, depending on the degree of plantar flexion in the brace ${ }^{5}$.

The estimated average Achilles tendon force at 1 year in this study was 2050 N (SD 330), and slightly higher than the results of a study that measured the Achilles tendon force on 8 healthy volunteers, using optical fibers ${ }^{6}$.

Our finding of a correlation between early walking speed and late calf muscle function was accidental, and must be interpreted with caution. However, it might reflect a connection between the patients' strive for physical activity and the final outcome, possibly via mechanostimulatory effects ${ }^{7}$. This might be related to the individuals' general attitude to physical exercise. There are also other observations that connect early findings with late results after Achilles tendon rupture: in 3 consecutive randomized trials it was found that the elastic modulus of the Achilles tendon 7 weeks after injury correlated with $\mathrm{HRI}$ at one year or later ${ }^{1,2,8,9}$. Pooling all data from these studies $(n=95)$ shows that $32 \%$ of the variance in final $H R I$ is explained by tendon elastic modulus at 7 weeks 
$(p=0.002$; data not shown). A recent randomized controlled study randomized 100 Achilles tendon rupture patients to surgical repair and early loading (starting 2 weeks after surgery), or neither surgical treatment nor early loading. There was no difference between the two groups at 1 year in terms of quality of life or functional results ${ }^{10}$. In the light of our findings this lack of an effect might have been partly caused by the inherent variation between patients in terms of inbrace-behaviour.

The low moment and thereby the weak forces in the Achilles tendon during bracing suggests that early weight bearing has little importance for mechanostimulation of healing. Still, early weight-bearing might encourage a more active lifestyle, which could facilitate later phases of mobilization and training.

To conclude, this study shows that a ruptured Achilles tendon will be virtually unloaded while the patient is walking in a cast. Moreover, calf muscle function 1 year after rupture seemed to be better in patients that were more active during their first 4 weeks after the rupture, as measured by the walking speed.

\section{References}

1. Schepull T, Aspenberg P. Early controlled tension improves the material properties of healing human achilles tendons after ruptures: a randomized trial. The American journal of sports medicine. 2013;41(11):2550-2557.

2. Schepull T, Kvist J, Andersson C, Aspenberg P. Mechanical properties during healing of Achilles tendon ruptures to predict final outcome: a pilot Roentgen stereophotogrammetric analysis in 10 patients. BMC Musculoskelet Disord. 2007;8:116.

3. Padulo J, Oliva F, Frizziero A, Maffulli N. Muscles, Ligaments and Tendons Journal. Basic principles and recommendations in clinical and field science research. MLTJ. 2013;4:250-252.

4. Finni T, Hodgson JA, Lai AM, Edgerton VR, Sinha S. Muscle synergism during isometric plantarflexion in achilles tendon rupture patients and in normal subjects revealed by velocityencoded cine phase-contrast MRI. Clinical biomechanics. 2006;21(1):67-74.

5. Akizuki KH, Gartman EJ, Nisonson B, Ben-Avi S, McHugh MP. The relative stress on the Achilles tendon during ambulation in an ankle immobiliser: implications for rehabilitation after Achilles tendon repair. Br J Sports Med. 2001;35(5):329-333; discussion 333-324.

6. Finni T, Komi PV, Lukkariniemi J. Achilles tendon loading during walking: application of a novel optic fiber technique. Eur $J$ Appl Physiol. 1998;77(3):289-291.

7. Andersson $T$, Eliasson $P$, Hammerman M, Sandberg O, Aspenberg $\mathrm{P}$. Low-level mechanical stimulation is sufficient to improve tendon healing in rats. J Appl Physiol. 2012;113(9):13981402.

8. Schepull T, Aspenberg P. Healing of human Achilles tendon ruptures: radiodensity reflects mechanical properties. Knee surgery, sports traumatology, arthroscopy: official journal of the ESSKA. 2013.

9. Schepull T, Kvist J, Aspenberg P. Early E-modulus of healing Achilles tendons correlates with late function: similar results with or without surgery. Scand J Med Sci Sports. 2012;22(1): 18-23.

10. Olsson N, Silbernagel KG, Eriksson BI, Sansone M, Brorsson A, Nilsson-Helander K, et al. Stable surgical repair with accelerated rehabilitation versus nonsurgical treatment for acute Achilles tendon ruptures: a randomized controlled study. Am J Sports Med. 2013;41(12):2867-2876. 